\title{
Calf hypertrophy and gastrocnemius MRI short tau inversion recovery (STIR) hyperintensity in a patient with asymptomatic hyperCKemia caused by caveolin-3 gene mutation
}

\author{
Dimitri Renard ${ }^{\mathrm{a}, \mathrm{b}, *}$, Florence Erny ${ }^{\mathrm{c}}$, Dominique Figarella-Branger ${ }^{\mathrm{d}}$, Martin Krahn ${ }^{\mathrm{e}, \mathrm{f}}$ \\ a Department of Neurology, CHU Nîmes, Hôpital Caremeau, Nîmes, France \\ ${ }^{\mathrm{b}}$ Centre de Référence des Maladies Neuromusculaires Grand Sud-Ouest, Nîmes, France \\ ' Department of Rhumatology, CHU Nîmes, Hôpital Caremeau, Nîmes, France \\ d Department of Pathology and Neuropathology, CHU Marseille, Hôpital La Timone, Marseille, France \\ • APHM, Département de Génétique Médicale, Hôpital Timone Enfants, 13385, Marseille, France \\ ${ }^{\mathrm{f}}$ Aix Marseille Université, INSERM UMR_S 910, GMGF, 13385, Marseille, France
}

Received 8 October 2015; received in revised form 27 November 2015; accepted 12 February 2016

Keywords: Calf hypertrophy; STIR; MRI; Muscle; Caveolin-3

We present a 16-year-old man with asymptomatic hyperCKemia fluctuating between 900 and $2400 \mathrm{IU} / \mathrm{L}$. His parents and his younger sister (13-year-old) were asymptomatic. Clinical examination showed no abnormalities apart of bilateral calf hypertrophy (calf circumference of $41 \mathrm{~cm}$ in both legs) (Fig. 1) [1]. Electromyography showed no abnormalities at rest, and small amplitude, brief, polyphasic action potentials, and early recruitment after muscle contraction in the proximal limb muscles. Lower limb muscle MRI (performed in the absence of unusual physical activity, exercise, or trauma preceding the MRI) confirmed calf hypertrophy and showed STIR hyperintensities in the bilateral medial gastrocnemius muscles (predominant on the right side) in the absence of abnormalities on T1-weighted imaging (Fig. 1), while the thighs did not show any abnormalities. Muscle biopsy of the right-sided medial gastrocnemius muscle showed myopathic changes (pathological fibre size variation, atrophic fibres, necrosis-regeneration, increased endomysial connective tissue, nuclear centralisation, core-like lesions) and immunohistochemical analyses showed normal expression of dystrophin, sarcoglycanes, dystroglycanes, merosin and nearly complete absence of caveolin-3 expression. Sequencing of the caveolin-3 gene (CAV3, NM_033337.2) identified a novel missense mutation in exon 1 [c.99C $>\mathrm{A}$; p.(Asn33Lys); not referenced in the Exome Aggregation Consortium Browser,

\footnotetext{
* Corresponding author. Department of Neurology, CHU Nîmes, Hôpital Caremeau, 4 Rue du Pr Debré, 30029 Nîmes Cedex 4, France. Tel.: +33 46668 32 61; fax: +33466684016.

E-mail address: dimitrirenard@hotmail.com (D. Renard).
}

http://exac.broadinstitute.org], corresponding to the same amino-acid change as the deleterious mutation c. $99 \mathrm{C}>\mathrm{G}$ [2]. Caveolin-3 deficiency is a rare, autosomal dominant, muscle disorder caused by $C A V 3$ mutations and consists of several clinical phenotypes including limb-girdle muscular dystrophy type $1 \mathrm{C}$, rippling muscle disease, distal myopathy, familial hypertrophic cardiopathy, and familial hyperCKemia. Few data exist on muscle MRI in CAV3-related muscle disorders, often reported as normal. Calf hypertrophy has been described in some rare reports in different clinical phenotypes related to CAV3 mutations [2,3]. In our patient, no inflammatory changes were seen on muscle biopsy to explain STIR hyperintensities. We assume that these STIR hyperintensities might correspond to muscle oedema. Clinical and radiological follow-up in our patient will show if the patient will develop clinical muscle symptoms over time and if calf hypertrophy and STIR hyperintensities will remain stable or if these "oedematose" changes are only a transitory state before evolving to fatty muscle atrophy typically seen in other muscle diseases (e.g. facioscapulohumeral muscular dystrophy) [4].

\section{References}

[1] McDowell MA, Fryar CD, Ogden CL, Flegal KM. Anthropometric reference data for children and adults: United States, 2003-2006. Natl Health Stat Report 2008;10:1-48.

[2] Sugie K, Murayama K, Noguchi S, et al. Two novel CAV3 gene mutations in Japanese families. Neuromuscul Disord 2004;14:810-14.

[3] Jacobi C, Ruscheweyh R, Vorgerd M, et al. Rippling muscle disease: variable phenotype in a family with five afflicted members. Muscle Nerve 2010;41:128-32.

[4] Friedman SD, Poliachik SL, Otto RK, et al. Longitudinal features of STIR bright signal in FSHD. Muscle Nerve 2014;49:257-60. 


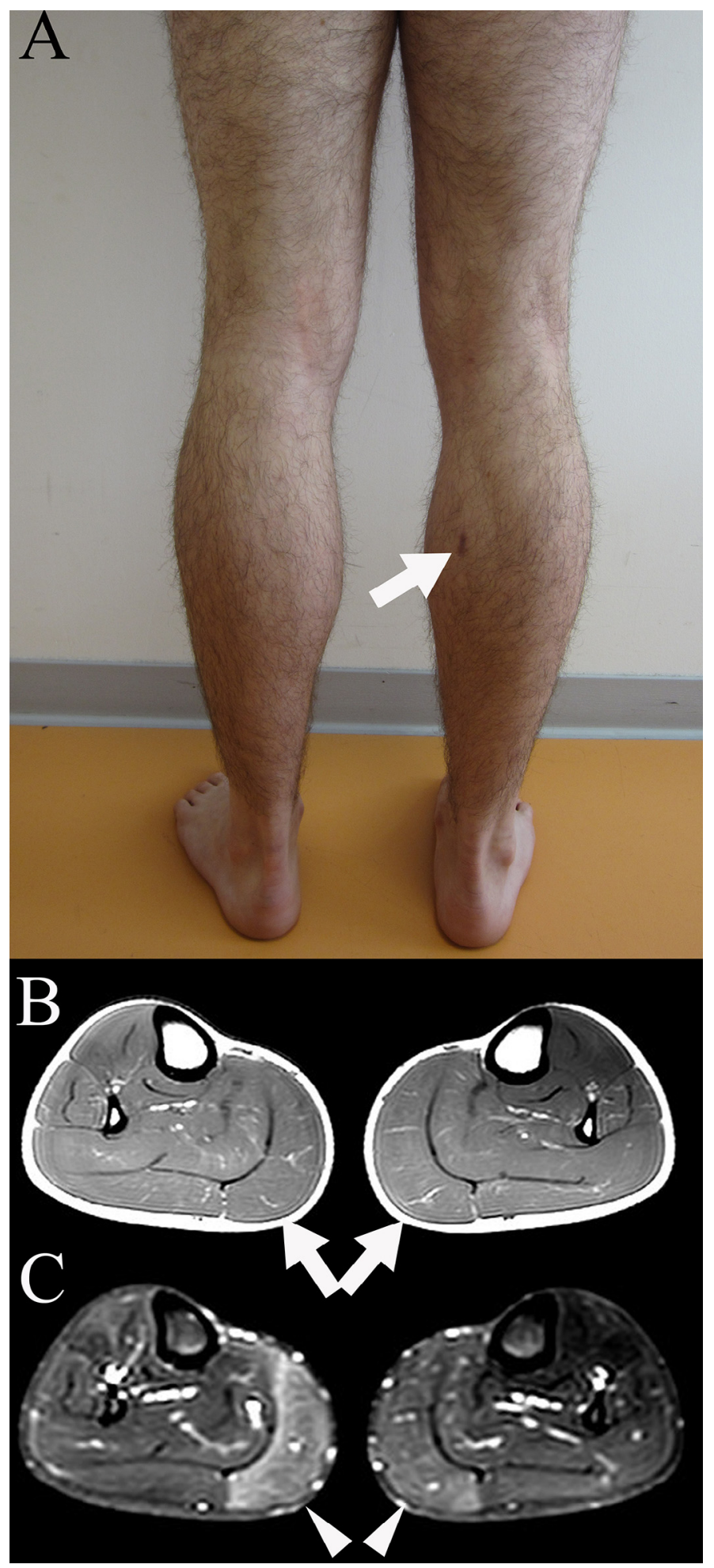

Fig. 1. Bilateral calf hypertrophy can be seen together with the skin lesion due to the right medial gastrocnemius muscle biopsy (A). Axial T1-weighted MRI imaging confirming bilateral calf hypertrophy in the absence of signal changes (B). STIR sequences showing bilateral hyperintensities in the medial gastrocnemius muscle predominant on the right side and very slight hyperintensities in the right-sided tibialis anterior muscle (C). 